ALEA, Lat. Am. J. Probab. Math. Stat. 18, 325-347 (2021)

DOI: 10.30757/ALEA.v18-15

\title{
The partial duplication random graph with edge deletion
}

\section{Felix Hermann and Peter Pfaffelhuber}

Technische Universität Berlin, Straße des 17. Juni 136,

10623 Berlin, Germany.

E-mail address: felix.hermann@tu-berlin.de

URL: http://page.math.tu-berlin.de/ hermann/

Albert-Ludwigs-Universität Freiburg,

Ernst-Zermelo-Straße 1,

79104 Freiburg, Germany.

E-mail address: peter.pfaffelhuber@stochastik.uni-freiburg.de

$U R L$ : https://www.stochastik.uni-freiburg.de/professoren/pfaffelhuber

\begin{abstract}
We study a random graph model in continuous time. Each vertex is partially copied with the same rate, i.e. an existing vertex is copied and every edge leading to the copied vertex is copied with independent probability $p$. In addition, every edge is deleted at constant rate, a mechanism which extends previous partial duplication models. In this model, we obtain results on the degree distribution, which shows a phase transition such that either - if $p$ is small enough - the frequency of isolated vertices converges to 1 , or there is a positive fraction of vertices with unbounded degree. We derive results on the degrees of the initial vertices as well as on the sub-graph of non-isolated vertices. In particular, we obtain expressions for the number of star-like subgraphs and cliques.
\end{abstract}

\section{Introduction}

Various random graph models have been studied in the last decades. Frequently, such models try to mimic the behavior of social networks (see e.g. Cooper and Frieze, 2003 and Barabási et al., 2002) or interactions within biological networks (see e.g. Wagner, 2001, Albert, 2005 and Jeong et al., 2000). For a general introduction to random graphs see the monographs Durrett (2007), van der Hofstad (2017) and references therein.

In this paper, we study and extend a duplication random graph model introduced and discussed in Bhan et al. (2002), Chung et al. (2003), Pastor-Satorras et al.

Received by the editors July 8th, 2019; accepted December 10th, 2020.

2010 Mathematics Subject Classification. 05C80 (Primary), 60K35 (Secondary).

Key words and phrases. Random graph, Degree distribution, Cliques. 
(2003), Chung et al. (2003), Bebek et al. (2006), Bebek et al. (2006), Hermann and Pfaffelhuber (2016), Jordan (2018) and, more recently, in Jacquet et al. (2020) and Frieze et al. (2020). In most applications, a vertex models a protein and an edge denotes some form of interaction; see e.g. Pastor-Satorras et al. (2003). Within the genome, the DNA encoding for a protein can be duplicated (which in fact is a long evolutionary process), such that the interactions of the copied protein are partially inherited to the copy; see Ohno (1970) for some more biological explanations. Within the random graph model, a vertex is $p$-copied, i.e. a new vertex is introduced and every edge of the parent vertex is independently copied with the same probability $p$. The idea behind this is to model protein-protein interactions, assuming that the ability to interact can be inherited from a parent protein with a fixed probability $p$.

In Pastor-Satorras et al. (2003), an extension of this model was suggested (but not studied further) where edges can be randomly removed from the random graph. Aiming for a closer look at this model, we extend the duplication random graph from above by introducing a rate $\delta$ at which each edge in the random graph is deleted. In biological terms, this corresponds to loss of interactive abilities due to mutation or deterioration; see e.g. Figure 3 in Wagner, 2001.

While in the previous literature no rigorous limit results were shown for the model without edge deletion, which we will call pure partial duplication model, Hermann and Pfaffelhuber (2016) have determined a critical parameter $p^{*} \approx 0.567143$, the unique solution of $p e^{p}=1$, below which approximately all vertices are isolated. Moreover, almost sure asymptotics and limit results for the number of $k$-cliques and $k$-stars in the random graph as well as for the degree of a fixed vertex were obtained. Recently, Jordan (2018) has shown that for $p<e^{-1}$ the degree distribution of the connected component, i.e. of the subgraph of non-isolated vertices, has a limit with tail behavior close to a power-law with exponent $\beta>2$ solving $\beta-3+p^{\beta-2}=0$ (cf. Jordan, 2018, Theorem 1(c)). This finding has been complemented by Jacquet et al. (2020) by some finer asymptotics. Extending the model for adding additional edges at random, Frieze et al. (2020) obtain results on the degree distribution and the degree of a fixed vertex. We also mention that Bienvenu et al. (2019) introduce a similar model for speciation. However, in their model, each birth of a new vertex is linked to removing another vertex, making the number of vertices in the network a constant.

In this paper for the model with edge deletion, we derive results on the degree distribution $F_{1}, F_{2}, \ldots$ of the full graph (see Theorem 2.4), the sub-graph of nonisolated vertices (see Proposition 2.6) as well as the number of star-like graphs, cliques, and degrees of initial vertices (see Theorem 2.9). The methods used to derive these results include branching processes with disasters $Z$ (see Section 3.1) and piecewise deterministic jump processes $X$ (see Section 3.2). For the former, note that the degree distribution is closely related via $\mathbb{P}\left(Z_{t}=k\right)=\mathbb{E}\left[F_{k}(t)\right]$ for all $k \geq 0$ (see (3.2)) - i.e. the expected degree distribution of the graph process equating to the distribution of $Z_{t}$. Such a connection to branching processes is as in Jordan (2018), but now $Z$ has additional deaths at rate $\delta$ - the rate of edge deletion. (Note that links between random graphs and branching processes frequently appear in the literature, e.g. van der Hofstad, 2017, Section 4.2 and Bollobás and Riordan, 2009.) For the latter, such branching processes, and therefore the degree distribution, can be studied by using piecewise-deterministic Markov 
jump processes, a tool which we introduce in Lemma 2.3; see also Section 3.1 for the connection to branching processes. The new connection of $X$ and $Z$ is via a duality relation (see (2.2)), which was already used in Hermann and Pfaffelhuber (2016), and proved extendable in various directions. Generalizing the limit results from Hermann and Pfaffelhuber, 2016, Lemma 3.3 for $X$ to a broader class of piecewisedeterministic Markov processes, we obtained general limit results for branching processes with disasters in several settings in Hermann and Pfaffelhuber (2020). In the present paper, we transfer these results to the degree distribution, generalizing the results of Theorem 2.7 in Hermann and Pfaffelhuber (2016) to the model with edge deletion; see Section 3. In particular, we derive a phase transition such that if $p$ is small, the fraction of vertices with positive degree vanishes. In this case we find that the sub-graph of non-isolated vertices is exponentially small, with two possible rates depending on $p$ and $\delta$; see Theorem 2.4. For larger $p$, a positive fraction of vertices is non-isolated, and their degree is unbounded. In Section 4, we prove Theorem 2.9 which states limit results for binomial moments of the degree distribution, cliques and the degree of a fixed node mainly by applying martingale theory, generalizing Theorems 2.9 and 2.14 in Hermann and Pfaffelhuber (2016).

\section{Model and main results}

After introducing the model (and its connection to a piecewise deterministic Markov process) in Section 2.1, we give our first main result, Theorem 2.4, on the number of non-isolated vertices, in Section 2.2. In Section 2.3, we discuss the (generating function of) the degree-distribution of the sub-graph of non-isolated vertices. Theorem 2.9 on certain graph functionals is contained in Subsection 2.4. Finally, we put our results in perspective to previous results in Subsection 2.5.

\subsection{The model and a piecewise deterministic Markov process.}

Definition 2.1 (Partial duplication graph process with edge deletion). Let $p \in$ $[0,1], \delta \geq 0$ and $G_{0}=\left(V_{0}, E_{0}\right)$ be a deterministic undirected graph without loops with vertex set $V_{0}=\left\{v_{1}, \ldots, v_{\left|V_{0}\right|}\right\}$ and non-empty edge set $E_{0}$. Let $P D(p, \delta)=$ $\left(G_{t}\right)_{t \geq 0}$ be the continuous-time graph-valued Markov process starting in $G_{0}$ and evolving in the following way:

- Every node $v \in V_{t}$ is $p$-partially duplicated (or $p$-copied for short) at rate $\kappa_{t}:=\left(\left|V_{t}\right|+1\right) /\left|V_{t}\right|$, i.e. a new node $v_{\left|V_{t}\right|+1}$ is added and for each $w \in V_{t}$ with $(v, w) \in E_{t}, v_{\left|V_{t}\right|+1}$ is connected to $w$ independently with probability $p$.

- Every edge in $E_{t}$ is removed at rate $\delta$.

Then, $P D(p, \delta)$ is a partial duplication graph process with edge deletion with initial graph $G_{0}$, edge-retaining probability $p$ and deletion rate $\delta$. Within $P D(p, \delta)$, we define the following quantities:

(1) Let $D_{i}(t):=\operatorname{deg}_{G_{t}}\left(v_{i}\right) \cdot \mathbf{1}_{\left\{i \leq\left|V_{t}\right|\right\}}$ be the degree of $v_{i}$, i.e. the number of its neighbors, at time $t$.

(2) Let $F(t):=\left(F_{k}(t)\right)_{k=0,1,2, \ldots}$ with $F_{k}(t):=\left|\left\{1 \leq i \leq\left|V_{t}\right|: D_{i}(t)=k\right\}\right| /\left|V_{t}\right|$ for $k=0,1,2, \ldots$ be the degree distribution at time $t$. Furthermore, let $F_{+}(t):=1-F_{0}(t)$ be the proportion of vertices of positive degree.

(3) For $k=1,2, \ldots$ let $B_{k}(t):=\sum_{\ell \geq k}\left(\begin{array}{l}\ell \\ k\end{array}\right) F_{\ell}(t)$ be the $k$ th binomial moment of the degree distribution. 
(4) For $k=1,2, \ldots$ let $C_{k}(t)$ be the number of $k$-cliques at time $t$, i.e. the number of complete sub-graphs of size $k$.

Remark 2.2. (1) It is often desirable to have a discrete-time graph valuedprocess where at each time $n$ the graph is of size $n$. For the $P D(p, \delta)$, such a discretization is also possible, but quite elaborate, since the number of edge deletions between two node additions follows a generalized negative hypergeometric distribution heavily depending on the number of edges and thus depending on the number of edges added with the latest node. In what follows, we will only discuss the continuous-time version.

(2) We choose the duplication rate $\kappa_{t}:=\left(\left|V_{t}\right|+1\right) /\left|V_{t}\right|$ in order to get a closed recurrence relation for the degree distribution; see Lemma 3.3. Alternatively, one could choose $\widetilde{\kappa}_{t}:=1$, i.e. all vertices are copied at unit rate. Since $\left|V_{t}\right| \sim V_{\infty} e^{t}$ (see Lemma 4.4) and hence $\int_{0}^{t} \kappa_{s}-\widetilde{\kappa}_{s} d s=\int_{0}^{t} \frac{1}{\left|V_{s}\right|} d s$ converges to a finite random variable, we conjecture that the random graph with our choice of $\kappa_{t}$ behaves the same qualitatively for $t \rightarrow \infty$ (i.e. underlying the same phase transitions as in Theorems 1 and 2). However, some limits depend on the intial graph (cf. $F_{0}$ in Theorem2.4(c)) and thus, since the choice of $\kappa_{t}$ influences the distribution of $G_{t}$ early on, quantitative differences are to be expected.

(3) For $k \in \mathbb{N}$ a $k$-star is a graph of $k+1$ nodes and $k$ edges, where one particular node, the center, is connected to each of the $k$ other nodes. Since each node of degree $\ell$ is the center of $\left(\begin{array}{l}\ell \\ k\end{array}\right)$ distinct $k$-stars, these deliver an alternative interpretation for the binomial moments: $\left|V_{t}\right| \cdot B_{k}(t)$ is equal to the total number of distinct $k$-stars contained as subgraphs in $G_{t}$. Hence, the $B_{k}(t)$ as well as the $C_{k}(t)$ can give an understanding of the topology of $G_{t}$. In fact, several functionals of interest can be expressed via $B_{k}$ and $C_{k}$ as the average degree in $G_{t}$ equates to $B_{1}(t)=C_{2}(t) /\left|V_{t}\right|$ while the transitivity ratio is given by $\frac{C_{3}(t)}{\left|V_{t}\right| B_{2}(t)}$.

Note that Hermann and Pfaffelhuber (2016) used the notation $S_{k}(t)$ for the factorial moments of the degree distribution giving $S_{k}(t) / k !=B_{k}(t)$.

In order to formulate our results, we need an auxiliary process, which is connected to $P D(p, \delta)$. It will appear below in Theorem 2.4.1 and in Proposition 2.6. The proof of the following Lemma is found in Section 3.2.

Lemma 2.3 (Connection of $P D(p, \delta)$ and a piecewise-deterministic Markov process). Let $X=\left(X_{t}\right)_{t \geq 0}$ be a Markov process on $[0,1]$ jumping at rate 1 from $X_{t}=x$ to $p x$, in between jumps evolving according to $\dot{X}_{t}=p X_{t}\left(1-X_{t}\right)-\delta X_{t}$. Furthermore, let

$$
H_{x}(t):=\sum_{k=0}^{\infty}(1-x)^{k} F_{k}(t)
$$

i.e. the probability generating function at $1-x$ of the degree distribution at time $t$. Then, for all $t \geq 0$ and $x \in[0,1]$, writing $\mathbb{E}_{x}[]:.=\mathbb{E}\left[. \mid X_{0}=x\right]$,

$$
\mathbb{E}\left[H_{x}(t)\right]=\mathbb{E}_{x}\left[H_{X_{t}}(0)\right]=\sum_{k=0}^{\infty} F_{k}(0) \cdot \mathbb{E}_{x}\left[\left(1-X_{t}\right)^{k}\right]
$$


2.2. Limits on the number of non-isolated vertices. Recall from the model with $\delta=0$ that there are at least three regimes: 1) If $p<1 / e\left(\right.$ or $\left.0>p-p \log \frac{1}{p}\right)$, Theorem 2.7 of Hermann and Pfaffelhuber (2016) shows that the frequency of isolated vertices converges to 1, and Theorem 2.1 of Jordan (2018) shows that the connected component converges to a graph with a power law distribution. 2) If $1 / e<p<p^{*}$, where $p^{*} \approx 0.567143$ is the unique solution of $p e^{p}=1$, (i.e. $p-p \log \frac{1}{p}>0>p-\log \frac{1}{p}$ ), the techniques of Jordan (2018) break down (see his Proposition 3.7), but still Theorem 2.7 of Hermann and Pfaffelhuber (2016) shows that the frequency of non-isolated vertices becomes negligible. 3) If $p>p^{*}$ (or $p-\log \frac{1}{p}>0$ ), the expected number of non-isolated vertices converges to a nontrivial fraction of the whole graph. Our first result, Theorem 2.4 below, extends this result to the case $\delta \geq 0$. The three cases (a), (b) and (c) of the following Theorem have 1), 2) and 3) as special cases for $\delta=0$. In Figure 2.1, we give an illustration of all cases.

For the formulation, we need some notation: For $X$ as in Lemma 2.3, we define

$$
c=\exp \left(-p \int_{0}^{\infty} \frac{\mathbb{E}_{1}\left[X_{s}^{2}\right]}{\mathbb{E}_{1}\left[X_{s}\right]} d s\right)
$$

Set $a_{t} \sim b_{t}$, if $a_{t} / b_{t} \stackrel{t \rightarrow \infty}{\longrightarrow} 1$, as well as $\prod_{\emptyset}=1$, and recall that $B_{k}(0)$ is the $k$ th binomial moment of the degree distribution of the initial graph, and in particular, $B_{1}(0)\left|V_{0}\right|$ is the initial number of edges.

Theorem 2.4 (Limit of the degree distribution). Let $p \in(0,1)$ and $\delta \geq 0$.

(a) If $\delta \geq p-p \log \frac{1}{p}$, then $F(t) \stackrel{t \rightarrow \infty}{\longrightarrow}(1,0,0, \ldots)$ almost surely with

$$
\mathbb{E}\left[F_{+}(t)\right] \sim c e^{-t(1+\delta-2 p)}
$$

where $c \in\left(0, B_{1}(0)\right)$ is given in $(2.3)$.

(b) If $p \geq e^{-1}$ and $p-p \log \frac{1}{p} \geq \delta \geq p-\log \frac{1}{p}$, then $F(t) \stackrel{t \rightarrow \infty}{\longrightarrow}(1,0,0, \ldots)$ almost surely with

$$
-\frac{1}{t} \log \mathbb{E}\left[F_{+}(t)\right] \stackrel{t \rightarrow \infty}{\longrightarrow} 1-\frac{1}{\gamma}(1+\log \gamma)
$$

where $\gamma=\log \frac{1}{p} /(p-\delta)$.

(c) If $p>\log \frac{1}{p}$ and $\delta<p-\log \frac{1}{p}$, then $F(t) \stackrel{t \rightarrow \infty}{\longrightarrow}\left(F_{0}, 0,0, \ldots\right)$ almost surely, where $F_{0}$ is non-deterministic and

$$
\mathbb{E}\left[F_{0}\right]=1-\left(1-\frac{\delta}{p}-\frac{1}{p} \log \frac{1}{p}\right) \sum_{k=1}^{\infty} B_{k}(0)(-1)^{k-1} \prod_{\ell=1}^{k-1}\left(1-\frac{\delta}{p}-\frac{1-p^{\ell}}{p \ell}\right) .
$$

Remark 2.5 (Interpretations). (1) Clearly, the quantity $F_{0}$ is increasing in $\delta$ and decreasing in $p$ (and $F_{+}$is decreasing in $\delta$ and increasing in $p$ ). See also the illustrations of Theorem 2.4 in Figure 2.1.

(2) For Theorem 2.4(c), we will see in the proof that the right hand side is the hitting probability of a stochastic process, and in particular is in $(0,1)$. This interpretation shows that $0<\mathbb{E}\left[F_{0}\right]<1$ as long as the initial graph is not trivial (i.e. $F_{0}(0)<1$ ).

(3) The asymptotics given in case $\delta \geq p-p \log \frac{1}{p}$ is more exact than the one given for $p-p \log \frac{1}{p} \geq \delta \geq p-\log \frac{1}{p}$ (in the sense that $-\frac{1}{t} \log \mathbb{E}\left[F_{+}(t)\right] \sim$ $1+\delta-2 p$ is a consequence of part 1 of the above Theorem). The reason is 
that we can give a formula for $c$ in this case, which does not carry over to (b); see the proof of Proposition 2.6.

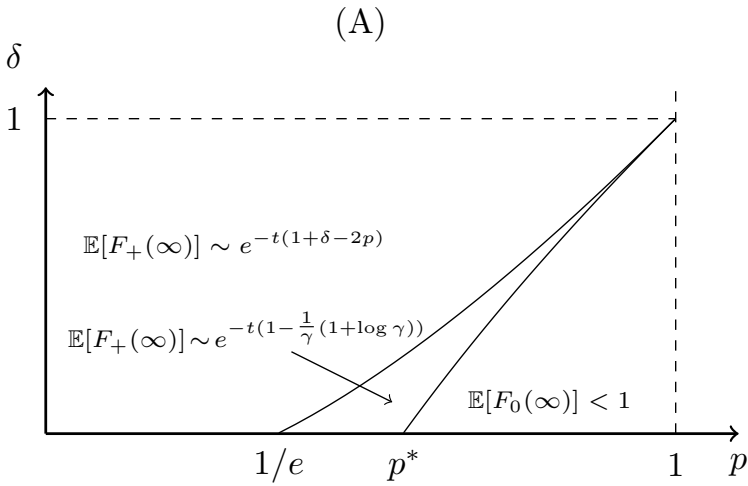

(B)

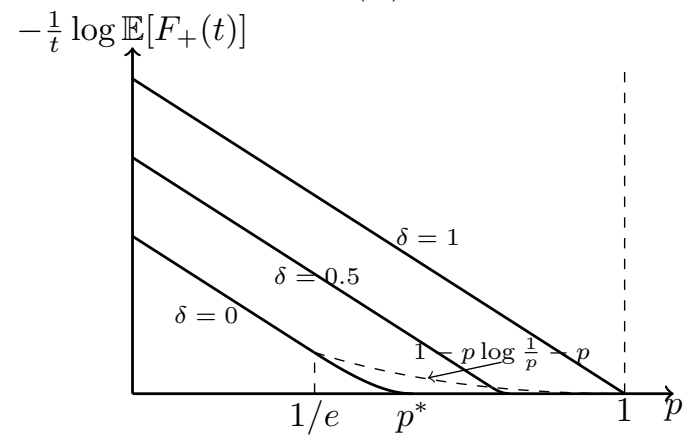

FIgURE 2.1.

Illustration of Theorem 2.4. In (A), the three cases are shown in the $p-\delta$-plane. In $(\mathrm{B})$, we draw the different exponential rates of decrease in $\mathbb{E}\left[F_{+}\right]$.

2.3. On the degree distribution of non-isolated vertices. In the case $\delta \geq p-\log \frac{1}{p}$, the frequency of isolated vertices converges to 1 . Hence, it is interesting to study the (degree distribution of the) sub-graph of non-isolated vertices. In order to do so, note that $F_{+}(t)=1-H_{1}(t)$, with $H$ from (2.1). Also note that, if at some time $t$ a duplication event is triggered, $1-H_{p}(t)$ denotes the probability that the new node is not isolated. Here, we aim for results on the asymptotics of the generating function of the degree distribution of the sub-graph of non-isolated vertices,

$$
h_{x}^{+}(t):=\frac{\sum_{k=1}^{\infty}(1-x)^{k} \mathbb{E}\left[F_{k}(t)\right]}{\mathbb{E}\left[F_{+}(t)\right]}=\frac{\mathbb{E}\left[H_{x}(t)-H_{1}(t)\right]}{\mathbb{E}\left[1-H_{1}(t)\right]}=1-\frac{\mathbb{E}\left[1-H_{x}(t)\right]}{\mathbb{E}\left[1-H_{1}(t)\right]}
$$

Using (2.2) and Bernoulli's formula we compute 


$$
\begin{aligned}
\mathbb{E}[1 & \left.-H_{x}(t)\right] \\
& =1-\sum_{k=0}^{\infty} F_{k}(0) \mathbb{E}_{x}\left[\left(1-X_{t}\right)^{k}\right]=1-\sum_{\ell=0}^{\infty} \mathbb{E}_{x}\left[X_{t}^{\ell}\right](-1)^{\ell} \sum_{k \geq \ell} F_{k}(0)\left(\begin{array}{l}
\ell \\
k
\end{array}\right) \\
& =1-\sum_{\ell=0}^{\infty}(-1)^{\ell} \mathbb{E}_{x}\left[X_{t}^{\ell}\right] \cdot B_{\ell}(0)=\sum_{\ell=1}^{\infty} B_{\ell}(0)(-1)^{\ell+1} \mathbb{E}_{x}\left[X_{t}^{\ell}\right] .
\end{aligned}
$$

As it turns out, we can control the right hand side as long as $\delta>p-p \log \frac{1}{p}$ (see Lemma 3.4). This immediately implies the following result:

Proposition 2.6 (Limit of degree distribution on the set of non-isolated vertices). Let $X$ be the process given in Lemma 2.3 and $\delta>p-p \log \frac{1}{p}$. Then, as $t \rightarrow \infty$,

$$
\mathbb{E}\left[1-H_{x}(t)\right] \sim \mathbb{E}_{x}\left[X_{t}\right] \cdot B_{1}(0), \quad \text { and therefore } \quad 1-h_{x}^{+}(t) \sim \frac{\mathbb{E}_{x}\left[X_{t}\right]}{\mathbb{E}_{1}\left[X_{t}\right]}
$$

For the other case, $p-\log \frac{1}{p}<\delta \leq p-p \log \frac{1}{p}$, the right hand side of (2.4) is not dominated by the $\mathbb{E}_{x}\left[X_{t}\right]$-term. Here, we can rather show that (see Section 3.4)

$$
\begin{aligned}
\frac{1}{t} \int_{0}^{t} \frac{\mathbb{E}_{x}\left[X_{s}^{k+1}\right]}{\mathbb{E}_{x}\left[X_{s}^{k}\right]} d s \stackrel{t \rightarrow \infty}{\longrightarrow} \frac{(p-\delta) k+p^{k}-(1+\log \gamma) / \gamma}{p k} \\
=\frac{\log \frac{1}{\gamma p^{k}}+\gamma p^{k}-1}{k \gamma p}=: c_{k}(p, \delta)
\end{aligned}
$$

where $\gamma=\log \frac{1}{p} /(p-\delta)$. However, to obtain a limit result in analogy to Proposition 2.6, we need this convergence not only to hold in the Cesaro-sense, but in the regular sense, i.e.

Conjecture 2.7. For $p \geq e^{-1}, p-\log \frac{1}{p}<\delta \leq p-p \log \frac{1}{p}$ and $k \geq 1$ it holds

$$
\frac{\mathbb{E}_{x}\left[X_{t}^{k+1}\right]}{\mathbb{E}_{x}\left[X_{t}^{k}\right]} \stackrel{t \rightarrow \infty}{\longrightarrow} c_{k}(p, \delta) \text {. }
$$

With this, inserting $\mathbb{E}_{x}\left[X_{t}^{\ell}\right]=\mathbb{E}_{x}\left[X_{t}\right] \prod_{k=1}^{\ell-1} \frac{\mathbb{E}_{x}\left[X_{t}^{k+1}\right]}{\mathbb{E}_{x}\left[X_{t}^{k}\right]}$, (2.4) immediately provides (a) and (b) of

Proposition 2.8. Assume that Conjecture 2.7 holds.

(a) If $p \geq e^{-1}, \delta=p-p \log \frac{1}{p}$, then $c_{1}(p, \delta)=0$ and $\mathbb{E}\left[1-H_{x}(t)\right] \sim \mathbb{E}_{x}\left[X_{t}\right]$. $B_{1}(0)$.

(b) If $p>e^{-1}, p-\log \frac{1}{p}<\delta<p-p \log \frac{1}{p}$, then

$$
\mathbb{E}\left[1-H_{x}(t)\right] \sim \mathbb{E}_{x}\left[X_{t}\right] \cdot \sum_{\ell=1}^{\infty} B_{\ell}(0)(-1)^{\ell+1} c_{1}(p, \delta) \cdots c_{\ell-1}(p, \delta) .
$$

In both cases, as $t \rightarrow \infty$,

$$
1-h_{x}^{+}(t) \sim \frac{\mathbb{E}_{x}\left[X_{t}\right]}{\mathbb{E}_{1}\left[X_{t}\right]} \sim x \exp \left(p \int_{0}^{\infty} \frac{\mathbb{E}_{1}\left[X_{s}^{2}\right]}{\mathbb{E}_{1}\left[X_{s}\right]}-\frac{\mathbb{E}_{x}\left[X_{s}^{2}\right]}{\mathbb{E}_{x}\left[X_{s}\right]} d s\right) .
$$

The last approximate equality is also shown in Section 3.4. Since the right hand side of (2.6) is non-trivial, this proposition implies - given that Conjecture 2.7 holds true - that the degree distribution of the sub-graph of non-isolated vertices converges to some non-trivial distribution. 
However, for a proof of Conjecture 2.7 or a closer analysis of the limits more insight into the process $X$ is necessary.

2.4. Limits of some graph-functionals. We now investigate the limiting behavior of certain functionals of the graph.

Theorem 2.9 (Binomial moments, cliques and degrees). As $t \rightarrow \infty$, the following statements hold almost surely:

(a) For $k=1,2, \ldots, e^{t \beta_{k}} B_{k}(t) \rightarrow B_{k}(\infty)$, where $B_{k}(\infty) \in \mathcal{L}^{1}$ and

$$
\beta_{k}= \begin{cases}1+\delta-2 p, & \text { if } \delta \geq p-\frac{p\left(1-p^{k-1}\right)}{k-1}, \\ 1+\delta k-p k-p^{k}, & \text { otherwise. }\end{cases}
$$

(b) For $k=2,3, \ldots, \exp \left(-t\left(k p^{k-1}-\delta\left(\begin{array}{l}k \\ 2\end{array}\right)\right)\right) C_{k}(t) \rightarrow C_{k}(\infty)$, where $C_{k}(\infty) \in$ $\mathcal{L}^{1}$

(i) If $C_{k}(0)>0$ and $\delta<2 p^{k-1} /(k-1)$, the convergence also holds in $\mathcal{L}^{1}$ and $\mathbb{P}\left(C_{k}(\infty)>0\right)>0$.

(ii) Otherwise, if $\delta \geq 2 p^{k-1} /(k-1), C_{k}(t)=0$ for all $t \geq T^{C_{k}}$ for some finite random variable $T^{C_{k}}$ and $\mathbb{P}\left(C_{k}(\infty)=0\right)=1$.

(c) For $i \leq\left|V_{0}\right|, e^{-t(p-\delta)} D_{i}(t) \rightarrow D_{i}(\infty)$, where $D_{i}(\infty) \in \mathcal{L}^{1}$. Moreover,

(i) if $D_{i}(0)>0$ and $\delta<p$, the convergence also holds in $\mathcal{L}^{r}$ for all $r \geq 1$ and

$$
\mathbb{E}\left[D_{i}(\infty)\right]=D_{i}(0)\left(1+\frac{p}{\left|V_{0}\right|}\right) .
$$

(ii) if $\delta \geq p, D_{i}(t)=0$ for all $t \geq T_{i}$ for some finite random variable $T_{i}$ and $\mathbb{P}\left(D_{i}(\infty)=0\right)=1$.

Remark 2.10 (Interpretations).

(1) For Theorem 2.9.(a), we have $\beta_{1}=1+\delta-2 p$ and for $\delta=0$, we have $\beta_{k}=1-p k-p^{k}, k=1,2, \ldots$ In all cases, we can also write $\beta_{k}=(1+\delta-$ $2 p) \wedge\left(1+\delta k-p k-p^{k}\right)$, which immediately shows that $\beta_{k}$ is continuous in $p$ and $\delta$. In addition, for $k=2,3, \ldots$, we find $p \geq \frac{p\left(1-p^{k-1}\right)}{k-1}$, i.e. we can choose $\delta \geq 0$ such that either of the two cases can in fact occur. Moreover, $\beta_{k} \leq \beta_{k-1}$, which can be seen as follows: First, note that $\frac{\left(1-p^{k-2}\right) /(k-2)}{\left(1-p^{k-1}\right) /(k-1)}=$ $\frac{\left(1+\cdots+p^{k-3}\right) /(k-2)}{\left(1+\cdots+p^{k-2}\right) /(k-1)} \geq 1$. So, if $\delta \geq p-\frac{p\left(1-p^{k-1}\right)}{k-1}$, both $\beta_{k-1}$ and $\beta_{k}$ do not depend on $k$ anyway. Then, if $p-\frac{p\left(1-p^{k-1}\right)}{k-1} \geq \delta \geq p-\frac{p\left(1-p^{k-2}\right)}{k-2}$, we have that

$$
\beta_{k-1}-\beta_{k}=1+\delta-2 p-\min \left(1+\delta-2 p, 1+\delta k-p k-p^{k}\right) \geq 0 .
$$

Finally, for $p-\frac{p\left(1-p^{k-2}\right)}{k-2} \geq \delta$, we have

$$
\begin{aligned}
\beta_{k-1}-\beta_{k} & =p-\delta-p^{k-1}+p^{k} \geq \frac{p\left(1-p^{k-1}\right)}{k-1}-p(1-p) p^{k-2} \\
& =p(1-p)\left(\frac{1+\cdots+p^{k-2}}{k-1}-p^{k-2}\right) \geq 0 .
\end{aligned}
$$

The fact that $\beta_{k-1} \geq \beta_{k}$ implies that there are much less star-like subgraphs with $k-1$ leaves than star-like subgraphs with $k$ leaves, $k=2,3, \ldots$ This can only be explained by nodes with high degree. 
(2) Noting that $B_{1}(t)=2|V(t)| \cdot C_{2}(t)$ and $\frac{1}{t} \log |V(t)| \stackrel{t \rightarrow \infty}{\longrightarrow} 1$, we see that the results in (a) and (b) imply the same growth rate for the number of edges.

(3) Interestingly, we find that $\delta \geq p$ implies that all vertices of the initial graph will eventually be isolated (i.e. have degree 0 ). However, the total number of edges, denoted by $C_{2}$, only dies out for $\delta \geq 2 p$. So, for $p<\delta<2 p$, all initial vertices become isolated, but are copied often enough such that the number of edges is positive for all times with positive probability.

(A)

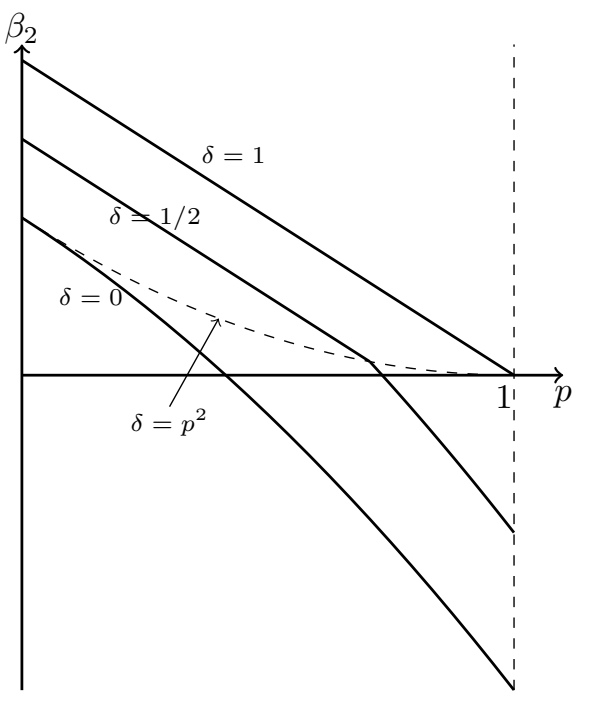

(B)

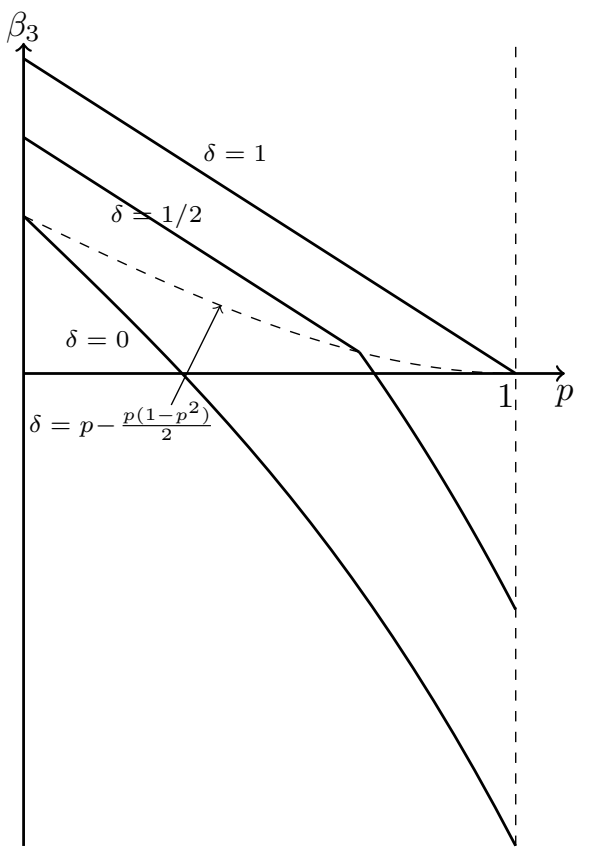

Figure 2.2.

Illustration of Theorem 2.4(a). We display the rates of decay of $\mathbb{E}\left[B_{k}(t)\right]$ for $k=2$ (A) and $k=3(\mathrm{~B})$.

2.5. Connection to previous work. Hermann and Pfaffelhuber (2016) analyzed the case $\delta=0$. Note that Theorem 2.7.1 in that paper is extended here by considering $\delta>0$ as well as giving precise exponential decay rates in Theorem 2.4(a) and 1(b). Moreover, recalling the connection $B_{k}(t)=S_{k}(t) / k$ ! mentioned in Remark 2.2.3, Theorem 2.7.2 is also generalized here to the case $\delta>0$ and further extended by the almost sure convergence of each component of the degree distribution in Theorem 2.4(c). The methods used in the proofs of Theorem 2.4 and Proposition 2.6 must be seen as extensions of tools used previously in Hermann and Pfaffelhuber (2016). In particular, we found that duplication graphs with edge deletion yield a similar connection to birth-death processes with disasters, defined in the next section. Such models can be studied using piecewiese-deterministic Markov processes; 
see Hermann and Pfaffelhuber (2020). Theorem 2.4 and Proposition 2.6 now essentially follow by combining Hermann and Pfaffelhuber, 2020, Corollaries 2.4 and 3.7 in the next section.

Theorem 2.9 in Hermann and Pfaffelhuber (2016) deals with cliques and $k$-stars in the case $\delta=0$ and is extended by Theorem 2.9. More precisely, since $\left|V_{t}\right| \sim e^{t}$, and Hermann and Pfaffelhuber (2016) treats the discrete-time model, we note that Theorem 2.9(1) of Hermann and Pfaffelhuber (2016) aligns with Theorem 2.9(b), but only gives $\mathcal{L}^{1}$ (rather than $\mathcal{L}^{2}$ )-convergence. In Theorem 2.9(2) of Hermann and Pfaffelhuber (2016), $S_{k}^{\circ}$, the number of $k$-stars in the network at time $t$ relative to the network size, was analyzed, which coincided with the factorial moments of the degree distribution. There, a $k$-star was not defined as a sub-graph of $G_{t}$, since it depended on the order of the nodes. $\left|V_{t}\right| \cdot B_{k}(t)$ now gives the number of starlike sub-graphs in the network at time $t$ consisting of $k+1$ nodes. Since the only difference between $S_{k}^{\circ}$ and $B_{k}$, as given in Theorem 2.9(a) is a factor of $k$ !, the results of Hermann and Pfaffelhuber (2016) easily apply also for $B_{k}$ if $\delta=0$. Theorem 2.14 of Hermann and Pfaffelhuber (2016) treats the degrees of initial vertices and thus can be compared to Theorem 2.9(c).

\section{Proof of Theorem 1}

Our analysis of the random graph $P D(p, \delta)$ is based on some main observations: First, the expected degree distribution can be represented by a birth-death process with binomial disasters $Z$, such that the distribution of $Z_{t}$ equals the expected degree distribution of $G_{t}$; see (3.2). Second, asymptotics for the survival probability of such processes were studied in Hermann and Pfaffelhuber (2020).

\subsection{Birth-death processes with disasters and p-jump processes.}

Definition 3.1. Let $b>0, d \geq 0$ and $p \in[0,1]$. Let $Z(b, d, p)=\left(Z_{t}\right)_{t \geq 0}$ be a continuous-time Markov process on $\mathbb{N}_{0}$ that evolves as follows: Given $Z_{0}=z$, the process jumps

- to $z+1$ at rate $b z$

- to $z-1$ at rate $d z$;

- to a binomially distributed random variable with parameters $z$ and $p$ at rate 1 .

Then we call $Z(b, d, p)$ a birth-death process subject to binomial disasters with birthrate $b$, death-rate $d$ and survival probability $p$.

Remark 3.2. (1) A birth-death process with binomial disasters, $Z(b, d, p)$ models the size of a population where each individual duplicates with rate $b$ and dies with rate $d$, subjected to binomial disasters at rate 1 . These disasters are global events that kill off each individual independently of each other with probability $1-p$, which generates the binomial distribution in the third part of Definition 3.1.

(2) Hermann and Pfaffelhuber (2020) provides several limit results for such branching processes with disasters. As reference for the following, let $Z=$ $Z(b, d, p)$ be as above. Then, Corollary 2.7 of Hermann and Pfaffelhuber (2020) states: 
(a) If $b-d \leq p \log \frac{1}{p}, Z$ goes extinct almost surely and

$$
\lim _{t \rightarrow \infty}-\frac{1}{t} \log \mathbb{P}\left(Z_{t}>0\right)=(1-p)-(b-d) .
$$

(b) If $p \log \frac{1}{p}<b-d \leq \log \frac{1}{p}, Z$ goes extinct almost surely and

$$
\lim _{t \rightarrow \infty}-\frac{1}{t} \log \mathbb{P}\left(Z_{t}>0\right)=1-\frac{b-d}{\log \frac{1}{p}}\left(1+\log \left(\frac{\log \frac{1}{p}}{b-d}\right)\right) .
$$

(c) If $b-d>\log \frac{1}{p}$, then $\mathbb{P}_{k}\left(\lim _{t \rightarrow \infty} Z_{t}=0\right)+\mathbb{P}_{k}\left(\lim _{t \rightarrow \infty} Z_{t}=\infty\right)=1$ and

$$
\mathbb{P}_{k}\left(\lim _{t \rightarrow \infty} Z_{t}=\infty\right)=\left(1-\frac{d+\log \frac{1}{p}}{b}\right) \sum_{\ell=1}^{k}\left(\begin{array}{l}
k \\
\ell
\end{array}\right)(-1)^{\ell-1} \prod_{m=1}^{\ell-1}\left(1-\frac{d m+\left(1-p^{m}\right)}{b m}\right) .
$$

By constructing a relationship between $P D(p, \delta)$ and $Z(p, \delta, p)$ in Lemma 3.3 , we are able to transfer these results to our duplication graph processes.

Lemma 3.3. Let $p \in(0,1), \delta \geq 0$ and recall $F_{k}(t)$ from Definition 2.1. As $h \rightarrow 0$, the entries $F_{k}$ of the degree distribution yield

$$
\begin{aligned}
\frac{1}{h} \mathbb{E}\left[F_{k}(t+h)-F_{k}(t) \mid G_{t}\right] & \\
= & -(1+p k+\delta k) F_{k}(t)+p(k-1) F_{k-1}(t)+\delta(k+1) F_{k+1}(t) \\
& +\sum_{\ell \geq k}\left(\begin{array}{l}
\ell \\
k
\end{array}\right) p^{k}(1-p)^{\ell-k} F_{\ell}(t)+o(1) .
\end{aligned}
$$

Moreover, recall $Z:=Z(p, \delta, p)$ from Definition 3.1 (i.e. the binomial distribution of the disasters has the birth rate as a parameter) and let $\mathbb{P}\left(Z_{0}=k\right)=F_{k}(0)$ for all $k$ be its initial distribution. Then, for all $t \geq 0$ and $k$, it holds

$$
\mathbb{P}\left(Z_{t}=k\right)=\mathbb{E}\left[F_{k}(t)\right],
$$

i.e. the distribution of $Z_{t}$ equals the expected degree distribution of $G_{t}$.

Proof: Letting $\Phi_{k}(t):=\left|V_{t}\right| F_{k}(t)$, the absolute number of nodes with degree $k$ at time $t$, we obtain for $h \rightarrow 0$ that

$$
\begin{aligned}
\frac{1}{h} \mathbb{E}\left[\Phi_{k}(t+h)-\right. & \left.\Phi_{k}(t) \mid G_{t}\right] \\
=- & \left(p k \kappa_{t}+\delta k\right) \Phi_{k}(t)+p(k-1) \kappa_{t} \Phi_{k-1}(t)+\delta(k+1) \Phi_{k+1}(t) \\
& +\sum_{\ell \geq k} \kappa_{t} \Phi_{\ell}(t)\left(\begin{array}{l}
\ell \\
k
\end{array}\right) p^{k}(1-p)^{\ell-k}+O(h),
\end{aligned}
$$

where the first term on the right hand side stands for the events at which a node can lose the degree $k$ by either obtaining a new neighbor (by one of its $k$ neighbors being copied, which happens with rate $k \kappa_{t}$, retaining at least the one relevant edge, which has probability $p$ ) or one of its $k$ edges being deleted, which happens at rate $\delta k$. The second and third terms describe the corresponding gain of a node with degree $k$ by analogous events. Finally, the sum equals the rate of a new node arising with degree $k$, which can only happen if a node of degree $\ell \geq k$ is copied (with rate $\kappa_{t} \Phi_{\ell}(t)$ ) and the copy retains exactly $k$ edges (which then has a binomial probability). 
Now, since $\left|V_{t}\right|$ only increases if a new node is added, i.e. on an event related to $\kappa_{t}$, it follows

$$
\begin{aligned}
& \frac{1}{h} \mathbb{E}\left[F_{k}(t+h)-F_{k}(t) \mid G_{t}\right] \\
& =\frac{1}{h} \mathbb{E}\left[\frac{\Phi_{k}(t+h)}{\left|V_{t+h}\right|}-\frac{\Phi_{k}(t)}{\left|V_{t+h}\right|} \mid G_{t}\right]+\frac{1}{h} \mathbb{E}\left[\frac{\Phi_{k}(t)}{\left|V_{t+h}\right|}-\frac{\Phi_{k}(t)}{\left|V_{t}\right|} \mid G_{t}\right] \\
& =\frac{\kappa_{t}}{\left|V_{t}\right|+1}\left(-p k \Phi_{k}(t)+p(k-1) \Phi_{k-1}(t)+\sum_{\ell \geq k} \Phi_{\ell}(t)\left(\begin{array}{l}
\ell \\
k
\end{array}\right) p^{k}(1-p)^{\ell-k}\right) \\
& \quad+\frac{-\delta k \Phi_{k}(t)+\delta(k+1) \Phi_{k+1}(t)}{\left|V_{t}\right|}+\kappa_{t}\left|V_{t}\right| \Phi_{k}(t)\left(\frac{1}{\left|V_{t}\right|+1}-\frac{1}{\left|V_{t}\right|}\right)+O(h) \\
& =-p k F_{k}(t)+p(k-1) F_{k-1}(t)+\sum_{\ell \geq k} F_{\ell}(t)\left(\begin{array}{l}
\ell \\
k
\end{array}\right) p^{k}(1-p)^{\ell-k} \\
& \quad-\delta k F_{k}(t)+\delta(k+1) F_{k+1}(t)-F_{k}(t)+O(h),
\end{aligned}
$$

and (3.1) holds. Computing the Kolmogorov forwards equation for $Z$ shows that for $k=0,1,2, \ldots$

$$
\begin{aligned}
\frac{d}{d t} \mathbb{P}\left(Z_{t}=k\right)=- & (1+p k+\delta k) \mathbb{P}\left(Z_{t}=k\right)+p(k-1) \mathbb{P}\left(Z_{t}=k-1\right) \\
& +\delta(k+1) \mathbb{P}\left(Z_{t}=k+1\right)+\sum_{\ell \geq k} \mathbb{P}\left(Z_{t}=\ell\right)\left(\begin{array}{l}
\ell \\
k
\end{array}\right) p^{k}(1-p)^{\ell-k},
\end{aligned}
$$

which is the same relation as (3.1) after taking expectation and letting $h \rightarrow 0$. This shows (3.2).

3.2. Properties of the piecewise deterministic jump process $X$. We have seen the connection of $P D(p, \delta)$ to a branching process with disasters in Lemma 3.3. Such branching processes are in turn closely connected to piecewise deterministic jump processes as in Lemma 2.3 (Hermann and Pfaffelhuber, 2020). Hence, we can now prove Lemma 2.3.

Proof of Lemma 2.3: Lemma 3.3 implies that $\mathbb{E}\left[H_{x}(t)\right]=\mathbb{E}\left[(1-x)^{Z_{t}}\right]$. Recognizing $\left(Z_{t}\right)=Z(p, \delta, p)$ as a homogeneous branching process with disasters $Z_{\lambda, q, 1, p}^{h}$ in the sense of Hermann and Pfaffelhuber, 2020, Definition 2.5, with death-rate $\lambda=p+\delta$ and offspring distribution $q=\left(q_{0}, 0, q_{2}, 0, \ldots\right)$ holding $q_{2}=\frac{p}{p+\delta}=1-q_{0}$, the result follows from Lemma 4.1 in Hermann and Pfaffelhuber (2020).

For the process $X$, we now obtain a property which is needed in the proofs of Theorem 2.4 and Proposition 2.6.

Lemma 3.4 (Moments of $X$ ). Let $X$ be as in Lemma 2.3. If $\delta>p-p \log \frac{1}{p}$, then $\mathbb{E}_{x}\left[X_{t}^{k}\right]=o\left(\mathbb{E}_{x}\left[X_{t}\right]\right)$ for all $k=2,3, \ldots$ and $\mathbb{E}_{x}\left[X_{t}\right] \sim c e^{-t(1+\delta-2 p)}$, where

$$
c:=x \cdot \exp \left(-p \int_{0}^{\infty} \frac{\mathbb{E}_{x}\left[X_{s}^{2}\right]}{\mathbb{E}_{x}\left[X_{s}\right]} d s\right) \in(0,1) .
$$

Proof: Recall $\gamma:=\log \frac{1}{p} /(p-\delta)$. Indeed, for $p-p \log \frac{1}{p}<\delta \leq p-p^{2} \log \frac{1}{p}$, such that $\gamma \in\left(p^{-1}, p^{-2}\right)$, it follows from Corollary 2.4 of Hermann and Pfaffelhuber (2020) that - independent of $x-$

$$
-\frac{1}{t} \log \left(\frac{\mathbb{E}_{x}\left[X_{t}^{2}\right]}{\mathbb{E}_{x}\left[X_{t}\right]}\right) \stackrel{t \rightarrow \infty}{\longrightarrow} 1-\frac{1+\log \gamma}{\gamma}-(1+\delta-2 p)=2 p \cdot c_{2}(p, \delta)>0
$$


On the other hand, if $\delta \geq p-p^{2} \log \frac{1}{p}$, the same corollary gives

$$
\begin{array}{r}
-\frac{1}{t} \log \left(\frac{\mathbb{E}_{x}\left[X_{t}^{2}\right]}{\mathbb{E}_{x}\left[X_{t}\right]}\right) \stackrel{t \rightarrow \infty}{\longrightarrow} 1+2 \delta-2 p-p^{2}-(1+\delta-2 p) \\
=\delta-p^{2} \geq p^{2}\left(\frac{1}{p}-1-\log \frac{1}{p}\right)>0 .
\end{array}
$$

In either case, there is an $\varepsilon>0$ such that $0<r(s):=\mathbb{E}_{x}\left[X_{s}^{2}\right] / \mathbb{E}_{x}\left[X_{s}\right]=O\left(e^{-\varepsilon s}\right)$ and it follows from (3.3)

$$
\mathbb{E}_{x}\left[X_{t}\right]=x \exp \left(-t(1+\delta-2 p)-p \int_{0}^{t} r(s) d s\right),
$$

conluding the proof.

3.3. Proof of Theorem 2.4. By (3.1) in Lemma 3.3, we get that, as $h \rightarrow 0$

$$
\begin{aligned}
\frac{1}{h} \mathbb{E}\left[F_{0}(t+h)-F_{0}(t) \mid G_{t}\right] & \rightarrow-F_{0}(t)+\delta F_{1}(t)+\sum_{\ell=0}^{\infty}(1-p)^{\ell} F_{\ell}(t) \\
& =\delta F_{1}(t)+\sum_{\ell=1}^{\infty}(1-p)^{\ell} F_{\ell}(t) \geq 0 .
\end{aligned}
$$

Hence, $\left(F_{0}(t)\right)_{t}$ is a bounded sub-martingale and converges almost surely and in $\mathcal{L}^{1}$. Consequently, the left hand side has to converge to 0 almost surely. Since $(1-p)^{\ell}$ is always positive, that can only be the case if $F_{\ell}(t) \rightarrow 0$ almost surely for all $\ell=1,2, \ldots$, which guarantees almost sure convergence of $F(t)$ to a vector of the form $F(\infty)=\left(F_{0}, 0,0, \ldots\right)$ in all cases.

Let $Z:=\left(Z_{t}\right)_{t \geq 0}:=Z(p, \delta, p)$ be as in Definition 3.1. We note that $\mathbb{E}\left[F_{+}(t)\right]=$ $\mathbb{P}\left(Z_{t}>0\right)$ by (3.2). For (a), we see from Lemma 2.3 and Lemma 3.4 that

$$
\begin{aligned}
\mathbb{E}\left[F_{+}(t)\right] & =1-\mathbb{E}\left[H_{1}(t)\right]=1-\sum_{k=0}^{\infty} F_{k}(0) \mathbb{E}_{1}\left[\left(1-X_{t}\right)^{k}\right] \\
& =\sum_{k=1}^{\infty} k F_{k}(0) \mathbb{E}_{1}\left[X_{t}\right]+o\left(\mathbb{E}_{1}\left[X_{t}\right]\right) \sim B_{1}(0) \cdot c e^{-t(1+\delta-2 p)}
\end{aligned}
$$

with $c$ as in (2.3). Moreover, (b) follows directly from Corollary 2.7 in Hermann and Pfaffelhuber (2020); see Remark 3.2.2. by setting $b=p$ and $d=\delta$. For (c), we again use Corollary 2.7 in Hermann and Pfaffelhuber (2020), but use in addition that

$$
\mathbb{E}\left[F_{0}(t)\right]=\mathbb{P}\left(Z_{t}=0\right)=\sum_{k=0}^{\infty} \mathbb{P}_{k}\left(Z_{t}=0\right) \cdot \mathbb{P}\left(Z_{0}=k\right),
$$

and $\sum_{k \geq \ell} F_{k}(0)\left(\begin{array}{c}k \\ \ell\end{array}\right)=B_{\ell}(0)$.

3.4. Proof of claims in Subsection 2.3. It remains to show (2.5) and the last equality in (2.6). Applying the generator of $X$ we see that its moments satisfy, for $k=$ $1,2, \ldots$,

$$
\begin{aligned}
\frac{d}{d t} \log \mathbb{E}_{x}\left[X_{t}^{k}\right] & =\frac{1}{\mathbb{E}_{x}\left[X_{t}^{k}\right]}\left(p^{k} \mathbb{E}_{x}\left[X_{t}^{k}\right]-\mathbb{E}_{x}\left[X_{t}^{k}\right]+(p-\delta) k \mathbb{E}_{x}\left[X_{t}^{k}\right]-p k \mathbb{E}_{x}\left[X_{t}^{k+1}\right]\right) \\
& =p^{k}+(p-\delta) k-1-\frac{p k \mathbb{E}_{x}\left[X_{t}^{k+1}\right]}{\mathbb{E}_{x}\left[X_{t}^{k}\right]}
\end{aligned}
$$


and thus, integrating, dividing by $-t$, and using Corollary 2.4 of Hermann and Pfaffelhuber (2020),

$$
\begin{aligned}
& \frac{1}{t} \int_{0}^{t} \mathbb{E}_{x}\left[X_{s}^{k+1}\right] / \mathbb{E}_{x}\left[X_{s}^{k}\right] d s=-\frac{1}{p k}\left(\frac{1}{t}\left(\log \mathbb{E}_{x}\left[X_{t}^{k}\right]-\log \left(x^{k}\right)\right)-p^{k}-(p-\delta) k+1\right) \\
& \stackrel{t \rightarrow \infty}{\longrightarrow} \frac{p-\delta+p^{k}-(1+\log \gamma) / \gamma}{p k}=c_{k}(p, \delta),
\end{aligned}
$$

which shows (2.5). Moreover, the last equality in (2.6) also follows from (3.3).

\section{Proof of Theorem 2.9}

The proof of Theorem 2.9, which is carried out in Section 4.4, will be based on the analysis of several martingales, which are derived in Proposition 4.5 in Section 4.3. In Section 4.2, we will analyze the total size of $G_{t}$.

4.1. Two auxiliary functions. We will need two specific functions in the sequel, which we now analyze.

Lemma 4.1. Let $p \in(0,1), \delta \geq 0$ and

$$
g: \begin{cases}{[0, \infty)} & \rightarrow \mathbb{R} \\ x & \mapsto 1+\delta x-p x-p^{x} .\end{cases}
$$

Then, $g$ is strictly concave and thus, $x \mapsto g(x) / x$ strictly decreases. Also, the following holds:

(1) If $\delta \geq p, g$ is strictly increasing and,

$$
g(x) \stackrel{x \rightarrow \infty}{\longrightarrow} \begin{cases}\infty, & \text { if } p<\delta, \\ 1, & \text { if } p=\delta .\end{cases}
$$

(2) If $p-\log \frac{1}{p}<\delta<p$,

$$
g \text { is }\left\{\begin{array}{l}
\text { strictly increasing on }(0, \xi), \\
\text { strictly decreasing on }(\xi, \infty)
\end{array}\right.
$$

for $\xi:=\log \gamma / \log \frac{1}{p}$ with $\gamma:=\log \frac{1}{p} /(p-\delta)$. The global maximum is $g(\xi)=$ $1-\frac{1}{\gamma}(1+\log \gamma)$

(3) If $\delta \leq p-\log \frac{1}{p}, g$ strictly decreases and its maximum is $g(0)=0$.

Proof: All results are straight-forward to compute. First, $g^{\prime}(x)=\delta-p+\log \frac{1}{p} \cdot p^{x}$ for all cases. Since the right hand side strictly increases, $g$ is strictly concave. Part 1. follows from the form of $g^{\prime}$. For part 3., we have that $g^{\prime}(x) \leq \delta-p+\log \frac{1}{p} \leq 0$, implying the result. Finally, for part 2., we have that $g^{\prime}(x)=0$ iff $p^{-x}=\log \frac{1}{p} /(p-$ $\delta)=\gamma$ iff $x=\log \gamma / \log \frac{1}{p}=\xi=\log \gamma /(\gamma(p-\delta))$ and the rest follows.

Lemma 4.2. Let $\Gamma$ denote the $\Gamma$-function, $r \in \mathbb{R}, g^{r}(n):=\frac{\Gamma(n+r)}{\Gamma(n)}$ and $n_{0} \geq$ $\max \{2,1-r\}$. Then, there are $0<c_{r} \leq 1<C_{r}<\infty$, such that

$$
c_{r} n^{r} \leq g^{r}(n) \leq C_{r} n^{r} \text { for all } n \geq n_{0} .
$$

Proof: First, we note that $g^{r}(n) \sim n^{r}$ as $n \rightarrow \infty$ (see e.g. 6.1.46. of Stein, 1970) and hence, the result follows. 
4.2. The size of the graph. For the asymptotics of the functionals of the random graph in Theorem 2.9 it will be helpful to understand the asymptotics of the process $\left(\left|V_{t}\right|\right)$. Here and below, we will frequently use the following well-known lemma.

Lemma 4.3. Let $X=\left(X_{t}\right)_{t \geq 0}$ be a Markov process with complete and separable state space $(E, r)$, and $f: E \rightarrow \mathbb{R}$ continuous and bounded and such that

$$
\lim _{h \rightarrow 0} \frac{1}{h} \mathbb{E}\left[f\left(X_{t+h}\right)-f\left(X_{t}\right) \mid X_{t}=x\right]=\lambda f(x), \quad x \in E
$$

for some $\lambda \in \mathbb{R}$, then $\left(e^{-t \lambda} f\left(X_{t}\right)\right)_{t \geq 0}$ is a martingale.

Proof: See Lemma 4.3.2 of Ethier and Kurtz (1986).

Lemma 4.4 (Graph size). Let $g^{r}(n):=\Gamma(n+r) / \Gamma(n)$. For all $r>-\left(\left|V_{0}\right|+1\right)$, the process $\left(e^{-t r} g^{r}\left(\left|V_{t}\right|+1\right)\right)_{t \geq 0}$ is a non-negative martingale. Moreover, there is a random variable $V_{\infty}$ such that the following holds:

$$
\begin{aligned}
& e^{-t}\left|V_{t}\right| \stackrel{t \rightarrow \infty}{\longrightarrow} V_{\infty} \text { almost surely and in } \mathcal{L}^{r} \text { for all } r \geq 1, \\
& e^{t} /\left|V_{t}\right| \stackrel{t \rightarrow \infty}{\longrightarrow} 1 / V_{\infty} \text { almost surely and in } \mathcal{L}^{r} \text { for } 1 \leq r<\left|V_{0}\right|+1 \\
& V_{\infty} \text { is } \Gamma\left(\left|V_{0}\right|+1,1\right) \text {-distributed. }
\end{aligned}
$$

Proof: Let $0<c_{r}<1<C_{r}<\infty$ be as in Lemma 4.2. The process $V:=\left(\left|V_{t}\right|\right)_{t \geq 0}$ is a Markov process which jumps from $v$ to $v+1$ at rate $v+1$. Setting $g^{r}(v)=$ $\Gamma(v+r) / \Gamma(v)$, we see that the process $\left(g^{r}\left(\left|V_{t}\right|+1\right)\right)_{t \geq 0}$ is well-defined and nonnegative if $\left|V_{t}\right|+1+r>0$ for all $t$, i.e. if $r>-\left(\left|V_{0}\right|+1\right)$. Then, as $h \rightarrow 0$,

$$
\begin{aligned}
\frac{1}{h} \mathbb{E}\left[g^{r}\left(V_{t+h}\right)-g^{r}\left(V_{t}\right) \mid V_{t}=v\right] & =(v+1)\left(g^{r}(v+2)-g^{r}(v+1)\right)+o(1) \\
& =(v+1) g^{r}(v+1)\left(\frac{v+1+r}{v+1}-1\right)+o(1) \\
& =r g^{r}(v+1)+o(1)
\end{aligned}
$$

and Lemma 4.3 implies that $\left(e^{-t r} g^{r}\left(\left|V_{t}\right|+1\right)\right)_{t \geq 0}$ is a (non-negative) martingale for all $r>-\left(\left|V_{0}\right|+1\right)$, and therefore $\mathcal{L}^{1}$-bounded. By the martingale convergence theorem, this martingale converges almost surely. Using (4.1), the martingale $\left(e^{-t} g^{1}\left(\left|V_{t}\right|+1\right)\right)_{t}=\left(e^{-t}\left(\left|V_{t}\right|+1\right)\right)_{t}$ is $\mathcal{L}^{r}$-bounded for every $r \geq 1$ and therefore converges in $\mathcal{L}^{r}$. Analogously, for $r=-1$, the martingale $\left(e^{t} g^{-1}\left(\left|V_{t}\right|+1\right)\right)_{t}=\left(e^{t} /\left|V_{t}\right|\right)_{t}$ is $\mathcal{L}^{r}$-bounded for $1 \leq r<\left|V_{0}\right|+1$ and converges in $\mathcal{L}^{r}$.

Noting that $\left(\left|V_{t}\right|+1\right)_{t>0}$ is a Yule-process starting in $\left|V_{0}\right|+1$, we have that $\left|V_{t}\right|+1$ is distributed as the sum of $\left|V_{0}\right|+1$ independent, geometrically distributed random variables with success probabilities $e^{-t}$ (see e.g. p. 109 of Athreya and Ney, 1972). Hence, as $t \rightarrow \infty$, we find that $e^{-t}\left|V_{t}\right|$ converges in distribution to the sum of $\left|V_{0}\right|+1$ independent, exponentially distributed random variables with unit rate. This is a $\Gamma\left(\left|V_{0}\right|+1,1\right)$ distribution.

4.3. Some martingales. Similarly to the discrete-time pure duplication graph in Hermann and Pfaffelhuber (2016) we obtain martingales for the functionals of $P D(p, \delta)$.

Proposition 4.5 (Martingales). Fix $k \geq 2$.

(1) Considering the function $g$ of Lemma 4.1, the following properties hold:

(a) If $g(1) \leq g(k),\left(e^{t g(1)} B_{k}(t)\right)_{t>0}$ is a martingale that almost surely converges to a limit $B_{k}(\infty) \in \mathcal{L}^{\overline{1}}$. 
(b) If $g(1)>g(k)$, there is a process $R_{k}(t)$ such that $\left(e^{t g(k)}\left(B_{k}(t)+\right.\right.$ $\left.\left.R_{k}(t)\right)\right)_{t \geq 0}$ is a positive martingale that almost surely converges to a limit $B_{k}(\infty) \in \mathcal{L}^{1}$ and $e^{t g(k)} R_{k}(t) \stackrel{t \rightarrow \infty}{\longrightarrow} 0$. In particular, $e^{t g(k)} B_{k}(t)$ $\rightarrow B_{k}(\infty)$ almost surely as $t \rightarrow \infty$.

Combining (a) and (b), we find $e^{t(g(1) \wedge g(k))} B_{k}(t) \stackrel{t \rightarrow \infty}{\longrightarrow} B_{k}(\infty) \in \mathcal{L}^{1}$.

(2) $\left(e^{-t\left(k p^{k-1}-1-\delta\left(\begin{array}{c}k \\ 2\end{array}\right)\right)} C_{k}(t) /\left|V_{t}\right|\right)_{t \geq 0}$ is a martingale that converges almost surely to a limit $\tilde{C}_{k}(\infty)$. If additionally $C_{k}(0)>0$ and $\delta<2 p^{k-1} /(k-1)$, the convergence also holds in $\mathcal{L}^{2}$.

(3) Let $i \leq\left|V_{0}\right|$. Then, $\left(e^{-t(p-\delta-1)} D_{i}(t) /\left|V_{t}\right|\right)_{t \geq 0}$ is a martingale that converges almost surely to a limit $\tilde{D}_{i}(\infty) \in \mathcal{L}^{1}$. Moreover,

$$
\mathbb{E}\left[e^{-t(p-\delta)} D_{i}(t)\right]=D_{i}(0)\left(1+\left(1-e^{-t}\right) \frac{p}{\left|V_{0}\right|}\right)
$$

and for $r \geq 2$, there is $C>0$, depending only on $r, p, \delta$ such that

$$
\mathbb{E}\left[\left(e^{-t(p-\delta)} D_{i}(t)\right)^{r}\right] \leq \mathbb{E}\left[\left(D_{i}(0)\right)^{r}\right]+C \int_{0}^{t} e^{-s(p-\delta)} \mathbb{E}\left[\left(e^{-s(p-\delta)} D_{i}(s)\right)^{r-1}\right] d s .
$$

Proof: 1 . Since the sum in $B_{k}(t)$ is almost surely finite for every $k$ and $t$, it follows for $h \rightarrow 0$ using equation (3.1), that

$$
\begin{gathered}
\frac{1}{h} \mathbb{E}\left[B_{k}(t+h)-B_{k}(t) \mid G_{t}\right] \\
=\sum_{\ell \geq k}\left(\begin{array}{l}
\ell \\
k
\end{array}\right)\left(-(1+p \ell+\delta \ell) F_{\ell}(t)+p(\ell-1) F_{\ell-1}(t)+\delta(\ell+1) F_{\ell+1}(t)\right. \\
\left.\quad+\sum_{m \geq \ell}\left(\begin{array}{c}
m \\
\ell
\end{array}\right) p^{\ell}(1-p)^{m-\ell} F_{m}(t)\right)+o(1) \\
=-B_{k}(t)+p(k-1) F_{k-1}(t)+\sum_{m \geq k} F_{m}(t) \sum_{\ell=k}^{m}\left(\begin{array}{c}
\ell \\
k
\end{array}\right)\left(\begin{array}{c}
m \\
\ell
\end{array}\right) p^{\ell}(1-p)^{m-\ell} \\
+\sum_{\ell \geq k} F_{\ell}(t)(\underbrace{-(p+\delta) \ell\left(\begin{array}{l}
\ell \\
k
\end{array}\right)+p \ell\left(\begin{array}{c}
\ell+1 \\
k
\end{array}\right)+\delta \ell\left(\begin{array}{c}
\ell-1 \\
k
\end{array}\right)}_{=: a(\ell, k)})+o(1) .
\end{gathered}
$$

Considering that $\left(\begin{array}{c}n+1 \\ m\end{array}\right)-\left(\begin{array}{c}n \\ m\end{array}\right)=\left(\begin{array}{c}n \\ m-1\end{array}\right)$ and $\frac{n}{m} \cdot\left(\begin{array}{c}n-1 \\ m-1\end{array}\right)=\left(\begin{array}{c}n \\ m\end{array}\right)$, we deduce

$$
\begin{aligned}
a(\ell, k) & =p \ell\left(\begin{array}{c}
\ell \\
k-1
\end{array}\right)-\delta \ell\left(\begin{array}{l}
\ell-1 \\
k-1
\end{array}\right)=(p-\delta) \ell\left(\begin{array}{l}
\ell-1 \\
k-1
\end{array}\right)+p \ell\left(\begin{array}{l}
\ell-1 \\
k-2
\end{array}\right) \\
& =(p-\delta) k\left(\begin{array}{l}
\ell \\
k
\end{array}\right)+p(k-1)\left(\begin{array}{c}
\ell \\
k-1
\end{array}\right),
\end{aligned}
$$

which implies that

$$
\begin{aligned}
& \frac{1}{h} \mathbb{E}\left[B_{k}(t+h)-B_{k}(t) \mid G_{t}\right] \\
& =-B_{k}(t)+p(k-1) B_{k-1}(t)+(p-\delta) k B_{k}(t) \\
& \quad+\sum_{m \geq k} F_{m}(t) \underbrace{\sum_{\ell=0}^{m-k}\left(\begin{array}{c}
m-k \\
\ell
\end{array}\right)\left(\begin{array}{c}
m \\
k
\end{array}\right) p^{\ell+k}(1-p)^{m-k-\ell}}_{=\left(\begin{array}{c}
m \\
k
\end{array}\right) p^{k}}+o(1)
\end{aligned}
$$




$$
\begin{aligned}
& =B_{k}(t)\left((p-\delta) k-\left(1-p^{k}\right)\right)+p(k-1) B_{k-1}(t)+o(1) \\
& =-g(k) B_{k}(t)+p(k-1) B_{k-1}(t)+o(1),
\end{aligned}
$$

recalling the function $g: x \mapsto 1+\delta x-p x-p^{x}$ from Lemma 4.1. In any case we see that $\left(e^{t g(1)} B_{1}(t)\right)_{t>0}$ is a non-negative martingale converging almost surely to a limit $B_{1}(\infty) \in \mathcal{L}^{1}$. For $k=2,3, \ldots$ let $g_{\min }(k):=\min _{1 \leq \ell \leq k} g(k)$ the running minimum of $g$. Then, there are two cases to consider:

1. $g(1) \leq g(k)$ : It holds by strict concavity of $g$ (see Lemma 4.1) that in this case $g(1)=g_{\min }(k)<g(\ell)$ for all $1<\ell<k$. Thus, letting

$$
\lambda_{m}^{k}:=\frac{g(1)}{g(k)} \prod_{\ell=m}^{k-1} \frac{p \ell}{g(\ell)-g(1)}, \quad m=2, \ldots, k
$$

and $\lambda_{1}^{k}:=1+\frac{1}{g(1)} p \lambda_{2}^{k}$, these coefficients are well-defined and positive. Considering the linear combination $Q_{k}(t):=\sum_{m=1}^{k} \lambda_{m}^{k} B_{m}(t)$ we obtain

$$
\begin{aligned}
\frac{1}{h} \mathbb{E}\left[Q_{k}(t+h)-Q_{k}(t) \mid G_{t}\right] & \\
=-g(1) B_{k}(t) & +\sum_{m=1}^{k-1} B_{m}(t)\left(-\lambda_{m}^{k} g(m)+\lambda_{m+1}^{k} p m\right)+o(1) \\
=-g(1) B_{k}(t) & +\sum_{m=2}^{k-1} B_{m}(t) \lambda_{m}^{k}\left(-g(m)+p m \frac{g(m)-g(1)}{p m}\right) \\
& \quad+B_{1}(t)\left(-g(1) \lambda_{1}^{k}+p \lambda_{2}^{k}\right)+o(1) \\
=-g(1) Q_{k}(t) & +o(1) .
\end{aligned}
$$

So now, $\left(e^{t g(1)} Q_{k}(t)\right)_{t \geq 0}$ is a non-negative martingale for every $k$. Since $B_{k}(t)$ can be represented as a linear combination of $\left(Q_{\ell}(t)\right)_{1 \leq \ell \leq k}$, also $\left(e^{t g(1)} B_{k}(t)\right)_{t \geq 0}$ has to be a non-negative martingale and thus converges to some $B_{k}(\infty) \in \mathcal{L}^{1}$.

2. $g(1)>g(k)$ : Here it holds by strict concavity of $g$ that $g(\ell)>g(k)=g_{\min }(k)$ for all $\ell=1, \ldots, k-1$. Hence

$$
\lambda_{m}^{k}:=\prod_{\ell=m}^{k-1} \frac{p \ell}{g(\ell)-g(k)}, \quad m=1, \ldots, k,
$$

are well-defined and positive. We compute analogously to the first case that, as $h \rightarrow 0$, with $Q_{k}(t):=\sum_{m=1}^{k} \lambda_{m}^{k} B_{m}$,

$$
\begin{aligned}
\frac{1}{h} & \mathbb{E}\left[Q_{k}(t+h)-Q_{k}(t) \mid G_{t}\right] \\
& =-g(k) B_{k}(t)+\sum_{m=1}^{k-1} B_{m}(t) \lambda_{m}^{k}\left(-g(m)+p m \frac{g(m)-g(k)}{p m}\right)+o(1) \\
& =-g(k) Q_{k}(t)+o(1) .
\end{aligned}
$$

Thus, $\left(e^{t g(k)} Q_{k}(t)\right)_{t \geq 0}$ is a non-negative martingale and has an almost sure limit $B_{k}(\infty) \in \mathcal{L}^{1}$. Moreover, for $\ell<k$, since $g(\ell)>g(k)$, we have that $e^{t g(k)} Q_{\ell}(t) \rightarrow 0$, so, writing $R_{k}(t)=Q_{k}(t)-B_{k}(t)$, we see that $R_{k}(t)=\sum_{\ell=1}^{k-1} \mu_{\ell}^{k} Q_{\ell}(t)$ for some $\mu_{1}^{k}, \ldots, \mu_{k-1}^{k}$ and $e^{t g(k)} R_{k}(t) \rightarrow 0$ and $e^{t g(k)} B_{k}(t) \rightarrow B_{k}(\infty)$ follows.

2. For the cliques fix $t \geq 0$ and let $N_{k}(v)$ for every node $v \in V_{t}$ denote the number 
of $k$-cliques that node is part of. Then, $\sum_{v \in V_{t}} N_{k}(v)=k C_{k}(t)$. Analogously define $M_{k}(e)$ as the number of cliques that the edge $e \in E_{t}$ is contained in, such that $\sum_{e \in E_{t}} M_{k}(e)=\left(\begin{array}{c}k \\ 2\end{array}\right) C_{k}(t)$. Also, let $\tilde{C}_{k}(t):=C_{k}(t) /\left|V_{t}\right|$. Note that for a new $k$ clique to arise, a node $v$ inside of such a clique has to be copied. Then, every of the $N_{k}(v)$ cliques $v$ is part of has a chance of $p^{k-1}$ that the copy obtains the $k-1$ edges it needs to form a new $k$-clique. Also, whenever an edge $e$ is deleted, all $M_{k}(e)$ $k$-cliques are destroyed. We deduce

$$
\begin{aligned}
\frac{1}{h} & \mathbb{E}\left[\tilde{C}_{k}(t+h)-\tilde{C}_{k}(t) \mid G_{t}\right] \\
& =\frac{\left|V_{t}\right|+1}{\left|V_{t}\right|} \sum_{v \in V_{t}}\left(\frac{C_{k}(t)+p^{k-1} N_{k}(v)}{\left|V_{t}\right|+1}-\tilde{C}_{k}(t)\right)-\delta \sum_{e \in E_{t}} \frac{M_{k}(e)}{\left|V_{t}\right|}+o(1) \\
& =\sum_{v \in V_{t}}\left(\tilde{C}_{k}(t)+\frac{p^{k-1} N_{k}(v)}{\left|V_{t}\right|}-\frac{\left|V_{t}\right|+1}{\left|V_{t}\right|} \tilde{C}_{k}(t)\right)-\frac{\delta}{\left|V_{t}\right|}\left(\begin{array}{c}
k \\
2
\end{array}\right) C_{k}(t)+o(1) \\
& =\tilde{C}_{k}(t)(\underbrace{k p^{k-1}-1-\delta\left(\begin{array}{c}
k \\
2
\end{array}\right)}_{=: q_{k}})+o(1) .
\end{aligned}
$$

This shows that $\left(e^{-t q_{k}} \cdot \tilde{C}_{k}(t)\right)_{t \geq 0}$ is a non-negative martingale and hence converges almost surely to an integrable random variable $\tilde{C}_{k}(\infty)$.

It remains to show the $\mathcal{L}^{2}$-convergence of the martingale $\left(e^{-t q_{k}} \tilde{C}_{k}(t)\right)_{t \geq 0}$ for $q_{k}+1>0$, i.e. $\delta<2 p^{k-1} /(k-1)$. This will be done by considering the number of (unordered) pairs of $k$-cliques, $C C_{k}(t):=\left(\begin{array}{c}C_{k}(t) \\ 2\end{array}\right)=\left(C_{k}(t)^{2}-C_{k}(t)\right) / 2$, and verifying that the process given by $\widetilde{C C}_{k}(t):=e^{-t \cdot 2 q_{k}} \frac{C C_{k}(t)}{\left|V_{t}\right|\left(\left|V_{t}\right|-1\right)}$ is $\mathcal{L}^{1}$-bounded, which implies $\mathcal{L}^{2}$-boundedness of the martingale $\left(e^{-t q_{k}} \cdot \tilde{C}_{k}(t)\right)_{t>0}$ and concludes the proof.

Let us denote by $C_{k, \ell}(t)$ the number of (unordered) pairs of $k$-cliques which have exactly $\ell$ shared vertices. Since the overlap of such a pair (i.e. the sub-graph both cliques have in common) is an $\ell$-clique with $\left(\begin{array}{l}\ell \\ 2\end{array}\right)$ edges, the number of edges making up the pair equals $2\left(\begin{array}{l}k \\ 2\end{array}\right)-\left(\begin{array}{l}\ell \\ 2\end{array}\right)$. Hence, arguing as in the proof of Theorem 2.9 in Hermann and Pfaffelhuber (2016), considering that (i) one new such pair arises if one of the $2(k-\ell)$ non-shared vertices is fully copied (probability $p^{k-1}$ ), and (ii) one new pair arises if one of the $\ell$ shared vertices is fully copied (probability $p^{2 k-\ell-1}$ ), by taking the copied node instead of the original one; in addition, there are two new pairs of $k$-cliques, one original and one copied, which share $\ell-1$ vertices, and (iii) if one of the $\ell$ shared vertices is chosen, but only one of the two cliques is fully copied (probability $2 p^{k-1}\left(1-p^{k-\ell}\right)$ ) one new pair of $k$-cliques arises, which shares $\ell-1$ vertices. In addition, such a pair will be destroyed if one of its edges is deleted, hence we deduce for $\ell \leq k-2$

$$
\begin{gathered}
\frac{1}{h} \mathbb{E}\left[C_{k, \ell}(t+h)-C_{k, \ell}(t) \mid G_{t}\right] \\
=\left(\left|V_{t}\right|+1\right) \cdot\left(\frac{2(k-\ell) p^{k-1}+\ell p^{2 k-\ell-1}}{\left|V_{t}\right|} C_{k, \ell}(t)+\frac{2(\ell+1) p^{k-1}}{\left|V_{t}\right|} C_{k, \ell+1}(t)\right) \\
-\delta \cdot\left(2\left(\begin{array}{l}
k \\
2
\end{array}\right)-\left(\begin{array}{l}
\ell \\
2
\end{array}\right)\right) \cdot C_{k, \ell}(t)+o(1),
\end{gathered}
$$

which implies for $\widetilde{C}_{k, \ell}(t):=e^{-t \cdot 2 q_{k}} \frac{C_{k, \ell}(t)}{\left|V_{t}\right|\left(\left|V_{t}\right|-1\right)}$, that

$$
\frac{1}{h} \mathbb{E}\left[\widetilde{C}_{k, \ell}(t+h)-\widetilde{C}_{k, \ell}(t) \mid G_{t}\right]
$$




$$
\begin{aligned}
& =-2 q_{k} \widetilde{C}_{k, \ell}(t)+e^{-t \cdot 2 q_{k}}\left(\left|V_{t}\right|+1\right) \\
& \cdot\left(\frac{\left(2(k-\ell) p^{k-1}+\ell p^{2 k-\ell-1}\right) C_{k, \ell}(t)+2(\ell+1) p^{k-1} C_{k, \ell+1}(t)}{\left|V_{t}\right| \cdot\left(\left|V_{t}\right|+1\right)\left|V_{t}\right|}\right. \\
& \left.+C_{k, \ell}(t) \cdot\left(\frac{1}{\left(\left|V_{t}\right|+1\right)\left|V_{t}\right|}-\frac{1}{\left|V_{t}\right|\left(\left|V_{t}\right|-1\right)}\right)\right) \\
& -2 \delta\left(\begin{array}{l}
k \\
2
\end{array}\right) \widetilde{C}_{k, \ell}(t)+\delta\left(\begin{array}{l}
\ell \\
2
\end{array}\right) \widetilde{C}_{k, \ell}(t)+o(1) \\
& =\widetilde{C}_{k, \ell}(t)\left(-2 q_{k}-2 \delta\left(\begin{array}{l}
k \\
2
\end{array}\right)+\delta\left(\begin{array}{l}
\ell \\
2
\end{array}\right)+\left(2(k-\ell) p^{k-1}+\ell p^{2 k-\ell-1}\right) \cdot \frac{\left|V_{t}\right|-1}{\left|V_{t}\right|}-2\right) \\
& +\widetilde{C}_{k, \ell+1}(t) \cdot 2(\ell+1) p^{k-1} \cdot \frac{\left|V_{t}\right|-1}{\left|V_{t}\right|}+o(1) \\
& \leq \widetilde{C}_{k, \ell}(t)\left(-2 \ell p^{k-1}+\ell p^{2 k-\ell-1}+\delta\left(\begin{array}{l}
\ell \\
2
\end{array}\right)\right)+\widetilde{C}_{k, \ell+1}(t) \cdot 2(\ell+1) p^{k-1}+o(1) \\
& =-\ell \widetilde{C}_{k, \ell}(t)\left(p^{k-1}\left(2-p^{k-\ell}\right)-\frac{\delta}{2}(\ell-1)\right)+2(\ell+1) p^{k-1} \widetilde{C}_{k, \ell+1}(t)+o(1) .
\end{aligned}
$$

Analogously, for $\ell=k-1$, additional pairs arise if a clique with $k$ vertices is completely copied (probability $p^{k-1}$ ), so

$$
\begin{aligned}
& \frac{1}{h} \mathbb{E}\left[\widetilde{C}_{k, k-1}(t+h)-\widetilde{C}_{k, k-1}(t) \mid G_{t}\right] \\
& \leq-(k-1) \widetilde{C}_{k, k-1}(t)\left(p^{k-1}(2-p)-\frac{\delta}{2}(k-2)\right) \\
& \quad+2 k p^{k-1} e^{-t \cdot 2 q_{k}} \cdot \frac{C_{k}(t)}{\left|V_{t}\right|\left(\left|V_{t}\right|-1\right)}+o(1) .
\end{aligned}
$$

Also, letting $\widehat{C}_{k}(t):=e^{-t \cdot 2 q_{k}} C_{k}(t) /\left(\left|V_{t}\right|\left(\left|V_{t}\right|-1\right)\right)$ and combining the calculation above with the one in (4.4), it follows that

$$
\begin{aligned}
& \frac{1}{h} \mathbb{E}\left[\widehat{C}_{k}(t+h)-\widehat{C}_{k}(t) \mid G_{t}\right] \\
& =-2 q_{k} \widehat{C}_{k}(t)+e^{-t \cdot 2 q_{k}}\left(\frac{k p^{k-1} C_{k}(t)}{\left|V_{t}\right|\left(\left|V_{t}\right|-1\right)} \cdot \frac{\left|V_{t}\right|-1}{\left|V_{t}\right|}+\frac{C_{k}(t)}{\left|V_{t}\right|}-C_{k}(t) \frac{\left|V_{t}\right|+1}{\left|V_{t}\right|\left(\left|V_{t}\right|-1\right)}\right) \\
& \quad-\delta\left(\begin{array}{c}
k \\
2
\end{array}\right) \widehat{C}_{k}(t)+o(1) \\
& \leq \widehat{C}_{k}(t)\left(-2 q_{k}+k p^{k-1}-2-\delta\left(\begin{array}{c}
k \\
2
\end{array}\right)\right)+o(1) \\
& =-\left(k p^{k-1}-\delta\left(\begin{array}{c}
k \\
2
\end{array}\right)\right) \widehat{C}_{k}(t)=-k \widehat{C}_{k}(t)\left(p^{k-1}\left(2-p^{0}\right)-\frac{\delta}{2}(k-1)\right)+o(1) .
\end{aligned}
$$

Now, since

$$
\delta<\frac{2 p^{k-1}}{k-1}=\min _{2 \leq m \leq k}\left\{\frac{2 p^{k-1}\left(2-p^{k-m}\right)}{m-1}\right\},
$$

the coefficients given by

$$
\lambda_{\ell}:=\prod_{m=1}^{\ell} \frac{2 p^{k-1}}{p^{k-1}\left(2-p^{k-m}\right)-\frac{\delta}{2}(m-1)}
$$


for $1 \leq \ell \leq k$ are well-defined and positive and we obtain for the linear combination

$$
R_{k}(t):=\widetilde{C}_{k, 0}(t)+\sum_{\ell=1}^{k-1} \lambda_{\ell} \widetilde{C}_{k, \ell}(t)+\lambda_{k} \widehat{C}_{k}(t)
$$

that

$$
\begin{aligned}
& \lim _{h \rightarrow 0} \frac{1}{h} \mathbb{E}\left[R_{k}(t+h)-R_{k}(t) \mid G_{t}\right] \\
& \quad \leq \sum_{\ell=1}^{k-1} \widetilde{C}_{k, \ell}(t) \cdot \ell\left(-\lambda_{\ell}\left(p^{k-1}\left(2-p^{k-\ell}\right)-\frac{\delta}{2}(\ell-1)\right)+\lambda_{\ell-1} 2 p^{k-1}\right) \\
& \quad \quad \quad \widehat{C}_{k}(t) \cdot k\left(-\lambda_{k}\left(p^{k-1}\left(2-p^{k-k}\right)-\frac{\delta}{2}(k-1)\right)+\lambda_{k-1} 2 p^{k-1}\right)=0 .
\end{aligned}
$$

Thus, $\left(R_{k}(t)\right)$ is a non-negative super-martingale, $\mathcal{L}^{1}$-bounded and, since $\lambda_{\text {min }}:=$ $\min \left(\{1\} \cup\left\{\lambda_{\ell} ; 1 \leq \ell \leq k\right\}\right)>0$ and $\widetilde{C C}_{k}(t) \leq R_{k}(t) / \lambda_{\min }$, the proof of 2 . is complete.

3. For the degree $D_{i}(t)$, we set $\left.\tilde{D}_{i}(t)=D_{i}(t)\right) /\left|V_{t}\right|$ and compute, as $h \rightarrow 0$,

$$
\begin{aligned}
& \frac{1}{h} \mathbb{E}\left[\tilde{D}_{i}(t+h)-\tilde{D}_{i}(t) \mid G_{t}\right] \\
& =\left(\left|V_{t}\right|+1\right)\left(\frac{p D_{i}(t)}{\left|V_{t}\right|}\left(\frac{D_{i}(t)+1}{\left|V_{t}\right|+1}-\frac{D_{i}(t)}{\left|V_{t}\right|}\right)+\left(1-\frac{p D_{i}(t)}{\left|V_{t}\right|}\right)\left(\frac{D_{i}(t)}{\left|V_{t}\right|+1}-\frac{D_{i}(t)}{\left|V_{t}\right|}\right)\right) \\
& \quad+\delta D_{i}(t)\left(\frac{D_{i}(t)-1}{\left|V_{t}\right|}-\frac{D_{i}(t)}{\left|V_{t}\right|}\right)+o(1) \\
& =\frac{D_{i}(t)}{\left|V_{t}\right|}\left(p \frac{\left|V_{t}\right|-D_{i}(t)}{\left|V_{t}\right|}-\left(1-\frac{p D_{i}(t)}{\left|V_{t}\right|}\right)-\delta\right)+o(1) \\
& =\tilde{D}_{i}(t)(p-\delta-1)+o(1) .
\end{aligned}
$$

Lemma 4.3 shows that $\left(e^{-t(p-\delta-1)} D_{i}(t) /\left|V_{t}\right|\right)_{t \geq 0}$ is a non-negative martingale, and hence converges almost surely. Furthermore, we write with $g^{r}(n):=\Gamma(n+r) / \Gamma(n)$

$$
\begin{aligned}
\frac{1}{h} \mathbb{E}\left[g^{r}\left(D_{i}(t+h)-g^{r}\left(D_{i}(t)\right) \mid G_{t}\right]\right. \\
=\left(\left|V_{t}\right|+1\right) \frac{p D_{i}(t)}{\left|V_{t}\right|}\left(g^{r}\left(D_{i}(t)+1\right)-g^{r}\left(D_{i}(t)\right)\right) \\
\quad+\delta D_{i}(t)\left(g^{r}\left(D_{i}(t)-1\right)-g^{r}\left(D_{i}(t)\right)\right)+o(1) \\
=g^{r}\left(D_{i}(t)\right)\left(p r \frac{\left|V_{t}\right|+1}{\left|V_{t}\right|}+\delta D_{i}(t)\left(\frac{D_{i}(t)-1}{D_{i}(t)+r-1}-1\right)\right)+o(1) \\
=g^{r}\left(D_{i}(t)\right) r(p-\delta)+g^{r}\left(D_{i}(t)\right) r\left(p\left(\frac{\left|V_{t}\right|+1}{\left|V_{t}\right|}-1\right)-\delta\left(\frac{D_{i}(t)}{D_{i}(t)+r-1}-1\right)\right) \\
\quad+o(1) \\
=g^{r}\left(D_{i}(t)\right) r(p-\delta)+g^{r}\left(D_{i}(t)\right) r\left(p \frac{1}{\left|V_{t}\right|}+\delta(r-1) \frac{1}{D_{i}(t)+r-1}\right) \\
\quad+o(1) .
\end{aligned}
$$


Letting $h \rightarrow 0$, this gives (4.2) for $r=1$, since

$$
\begin{aligned}
\mathbb{E}\left[e^{-t(p-\delta)} D_{i}(t)\right] & =D_{i}(0)+\int_{0}^{t} p \mathbb{E}\left[e^{-s(p-\delta)} D_{i}(s) /\left|V_{s}\right|\right] d s \\
& =D_{i}(0)+\int_{0}^{t} p e^{-s} D_{i}(0) /\left|V_{0}\right| d s
\end{aligned}
$$

where in the last step we used the martingale $\left(e^{-t(p-\delta-1)} D_{i}(t) /\left|V_{t}\right|\right)_{t \geq 0}$. Moreover, since $\frac{g^{r}\left(D_{i}(t)\right)}{D_{i}(t)+r-1}=g^{r-1}\left(D_{i}(t)\right),(4.5)$ gives for some $C>0$, depending on $r, p, \delta$

$$
\frac{d}{d t} \mathbb{E}\left[e^{-\operatorname{tr}(p-\delta)} g^{r}\left(D_{i}(t)\right)\right] \leq C \cdot \mathbb{E}\left[e^{-\operatorname{tr}(p-\delta)} g^{r-1}\left(D_{i}(t)\right)\right],
$$

and (4.3) follows with Lemma 4.2 and integration.

4.4. Proof of Theorem 2.9. (a). Recalling the function $g$ from Lemma 4.1, we note that (see also Remark2.10.1 for the second equality)

$$
\beta_{k}=(1+\delta-2 p) \wedge 1+(\delta-p) k-p^{k}=g(1) \wedge g(k) .
$$

Hence, Lemma 4.5.1 shows that $e^{t \beta_{k}} B_{k}(t)$ is non-negative and converges to some $B_{k}(\infty) \in \mathcal{L}^{1}$. So, (a) follows.

(b) We combine Lemma 4.5 .2 (recall the random variable $\tilde{C}_{k}(\infty)$ ) with the almost sure convergence $e^{-t} V_{t} \stackrel{t \rightarrow \infty}{\longrightarrow} V_{\infty}$ from Lemma 4.4. In all cases, we have that

$$
\begin{aligned}
\exp ( & \left.-t\left(k p^{k-1}-\delta\left(\begin{array}{l}
k \\
2
\end{array}\right)\right)\right) C_{k}(t) \\
& =\exp \left(-t\left(k p^{k-1}-1-\delta\left(\begin{array}{l}
k \\
2
\end{array}\right)\right)\right) C_{k}(t) /\left|V_{t}\right| \cdot e^{-t}\left|V_{t}\right| \\
& \stackrel{t \rightarrow \infty}{\longrightarrow} \tilde{C}_{k}(\infty) \cdot V_{\infty}=: C_{k}(\infty),
\end{aligned}
$$

where $V_{\infty}>0$ almost surely.

If $\delta \geq 2 p^{k-1} /(k-1)$, it is $k p^{k-1}-\delta\left(\begin{array}{c}k \\ 2\end{array}\right) \leq 0$ and the convergence can only hold if $C_{k}(t) \stackrel{t \rightarrow \infty}{\longrightarrow} 0$ almost surely. Since $C_{k}(t) \in \mathbb{N}_{0}$, the first hitting time $T^{C_{k}}$ of 0 has to be finite. On the other hand, for $\delta<2 p^{k-1} /(k-1)$, combining the $\mathcal{L}^{2}$-convergences in Lemma 4.5.2 and Lemma 4.4 we obtain that the convergence in (4.6) also holds in $\mathcal{L}^{1}$. Since $\left(e^{-t\left(k p^{k-1}-1-\delta\left(\begin{array}{c}k \\ 2\end{array}\right)\right)} C_{k}(t) /\left|V_{t}\right|\right)$ is an $\mathcal{L}^{2}$-convergent and thus uniformly integrable martingale, $\mathbb{P}\left(C_{k}(\infty)>0\right)=\mathbb{P}\left(\tilde{C}_{k}(\infty)>0\right)>0$.

(c) Finally, fix $i \in\left\{1, \ldots,\left|V_{0}\right|\right\}$. Again, we combine Lemma 4.5.3 (recall the random variable $\left.\tilde{D}_{i}(\infty)\right)$ with the almost sure convergence $e^{-t} V_{t} \stackrel{t \rightarrow \infty}{\longrightarrow} V_{\infty}$ from Lemma 4.4. In all cases, we have that

$$
e^{-t(p-\delta)} D_{i}(t)=e^{-t(p-\delta-1)} \frac{D_{i}(t)}{\left|V_{t}\right|} e^{-t}\left|V_{t}\right| \stackrel{t \rightarrow \infty}{\longrightarrow} \tilde{D}_{i}(\infty) \cdot V_{\infty}=: D_{i}(\infty)
$$

If $\delta<p$, we find by (4.2) that $\left(e^{-t(p-\delta)} D_{i}(t)\right)_{t \geq 0}$ is $\mathcal{L}^{1}$-bounded. Then, inductively using (4.3) shows that $\left(e^{-t(p-\delta)} D_{i}(t)\right)_{t \geq 0}$ is $\mathcal{L}^{r}$-bounded for all $r \geq 1$. In particular, this implies that the convergence in (4.7) also holds in $\mathcal{L}^{r}$ for all $r \geq 1$. This gives convergence of first moments, and (2.7) follows by taking $t \rightarrow \infty$ in (4.2).

If $\delta \geq p$, the almost sure convergence in (4.7) implies, since $D_{i}(t) \in \mathbb{N}_{0}$, that $D_{i}(\infty)=0$, so there must be a finite hitting time $T_{i}$ of 0 . 


\section{Acknowledgements}

We would like to thank an anonymous referee for helping to significantly improve the manuscript and Monika Hattenbach for her help with typesetting.

\section{References}

Albert, R. Scale-free networks in cell biology. Journal of Cell Science, 118 (21), 4947-4957 (2005). DOI: 10.1242/jcs.02714.

Athreya, K. B. and Ney, P. E. Branching processes. Springer-Verlag, New YorkHeidelberg (1972). MR0373040.

Barabási, A. L., Jeong, H., Néda, Z., Ravasz, E., Schubert, A., and Vicsek, T. Evolution of the social network of scientific collaborations. Phys. A, 311 (3-4), 590-614 (2002). MR1943379.

Bebek, G., Berenbrink, P., Cooper, C., Friedetzky, T., Nadeau, J., and Sahinalp, S. C. The degree distribution of the generalized duplication model. Theoret. Comput. Sci., 369 (1-3), 239-249 (2006). MR2277572.

Bebek, G., Berenbrink, P., Cooper, C., Nadeau, J., and Sahinalp, S. C. Improved duplication models for proteome network evolution. Systems Biology and Regulatory Genomics, 4023, 119-137 (2006).

Bhan, A., Galas, D. J., and Dewey, T. G. A duplication growth model of gene expression networks. Bioinformatics, 18 (11), 1486-1493 (2002). DOI: 10.1093/bioinformatics/18.11.1486.

Bienvenu, F., Débarre, F., and Lambert, A. The split-and-drift random graph, a null model for speciation. Stochastic Process. Appl., 129 (6), 2010-2048 (2019). MR3958422.

Bollobás, B. and Riordan, O. Random graphs and branching processes. In Handbook of large-scale random networks, volume 18 of Bolyai Soc. Math. Stud., pp. 15-115. Springer, Berlin (2009). MR2582386.

Chung, F., Lu, L., Dewey, T., and Galas, D. Duplication models for biological networks. J. Comput. Biol., 10 (5), 677-687 (2003). DOI: 10.1089/106652703322539024.

Cooper, C. and Frieze, A. A general model of web graphs. Random Structures Algorithms, 22 (3), 311-335 (2003). MR1966545.

Durrett, R. Random graph dynamics, volume 20 of Cambridge Series in Statistical and Probabilistic Mathematics. Cambridge University Press, Cambridge (2007). ISBN 978-0-521-86656-9; 0-521-86656-1. MR2271734.

Ethier, S. N. and Kurtz, T. G. Markov processes. Characterization and convergence. Wiley Series in Probability and Mathematical Statistics: Probability and Mathematical Statistics. John Wiley \& Sons, Inc., New York (1986). ISBN 0471-08186-8. MR838085.

Frieze, A., Turowski, K., and Szpankowski, W. Degree distribution for duplicationdivergence graphs: large deviations, pp. 226-237. Springer, Cham (2020). DOI: 10.1007/978-3-030-60440-0.

Hermann, F. and Pfaffelhuber, P. Large-scale behavior of the partial duplication random graph. ALEA Lat. Am. J. Probab. Math. Stat., 13 (2), 687-710 (2016). MR3531396. 
Hermann, F. and Pfaffelhuber, P. Markov branching processes with disasters: extinction, survival and duality to $p$-jump processes. Stochastic Process. Appl., 130 (4), 2488-2518 (2020). MR4074709.

Jacquet, P., Tutowski, K., and Szpankowski, W. Power-Law Degree Distribution in the Connected Component of a Duplication Graph. In Drmota, M. and Heuberger, C., editors, 31st International Conference on Probabilistic, Combinatorial and Asymptotic Methods for the Analysis of Algorithms (AofA 2020), volume 159 of Leibniz International Proceedings in Informatics (LIPIcs), pp. 16:1-16:14. Schloss Dagstuhl-Leibniz-Zentrum für Informatik, Dagstuhl, Germany (2020). ISBN 978-3-95977-147-4. DOI: 10.4230/LIPIcs.AofA.2020.16.

Jeong, H., Tombor, B., Albert, R., Oltvai, Z. N., and Barabási, A.-L. The largescale organization of metabolic networks. Nature, 407 (6804), 651-654 (2000). DOI: $10.1038 / 35036627$.

Jordan, J. The connected component of the partial duplication graph. ALEA Lat. Am. J. Probab. Math. Stat., 15 (2), 1431-1445 (2018). MR3882189.

Ohno, S. Evolution by Gene Duplications. Springer, Berlin, Heidelberg (1970). ISBN 978-3-642-86661-6, 978-3-642-86659-3. DOI: 10.1007/978-3-642-86659-3.

Pastor-Satorras, R., Smith, E., and Solé, R. V. Evolving protein interaction networks through gene duplication. J. Theoret. Biol., 222 (2), 199-210 (2003). MR2070214.

Stein, J. Table errata: Handbook of mathematical functions with formulas, graphs, and mathematical tables (Nat. Bur. Standards, Washington, D.C., 1964) edited by Milton Abramowitz and Irene A. Stegun. Math. Comp., 24 (110), 503 (1970). MR415962.

van der Hofstad, R. Random graphs and complex networks. Vol. 1. Cambridge Series in Statistical and Probabilistic Mathematics, [43]. Cambridge University Press, Cambridge (2017). ISBN 978-1-107-17287-6. MR3617364.

Wagner, A. The yeast protein interaction network evolves rapidly and contains few redundant duplicate genes. Mol. Biol. Evol., 18 (7), 1283-1292 (2001). DOI: 10.1093/oxfordjournals.molbev.a003913. 